https://helda.helsinki.fi

\title{
Cutaneous and systemic complications associated with tattooing
}

\section{Kluger, Nicolas}

2016-06

Kluger , N 2016 , ' Cutaneous and systemic complications associated with tattooing ' , La

Presse Médicale , vol. 45 , no. 6 , pp. 567-576 . https://doi.org/10.1016/j.lpm.2016.02.016

http://hdl.handle.net/10138/224096

https://doi.org/10.1016/j.Ipm.2016.02.016

publishedVersion

Downloaded from Helda, University of Helsinki institutional repository.

This is an electronic reprint of the original article.

This reprint may differ from the original in pagination and typographic detail.

Please cite the original version. 


\section{Q Crossark Cutaneous and systemic complications associated with tattooing}

Nicolas Kluger

University of Helsinki, Helsinki University Central Hospital, dermatology, allergology and venereology, Meilahdentie 2, P.0. Box 160, Fl-00029 HUS, Finland

nicolas.kluger@hus.fi

\section{Key points}

Tattooing can result in a wide variety of complications, whose prevalence and incidence remain still unclear. Hypersensitivity reactions (or allergies) to tattoo pigments are currently the most common complication on a tattoo, however they are not predictable.

Infections are nowadays directly related to the lack of asepsis and hygiene during the tattooing procedure or during the healing phase.

Patients with a known cutaneous disease should be warned of a potential risk of localization of their disease to the tattoo.

A skin eruption restricted to a tattoo may reveal sarcoidosis.

Patients with chronic conditions and/or impaired immunity should discuss with their physician about the possibility and when to have a tattoo.

\section{Points essentiels}

Complications cutanées et systémiques associées au tatouage

Le tatouage peut être associé avec un grand nombre de complications, dont la prévalence et l'incidence restent encore mal connues à ce jour.

L'allergie à l'encre de tatouage (le rouge principalement) est la principale complication à craindre, mais elle est actuellement encore imprévisible.

Les infections sur tatouage sont directement dues au manque d'asepsie ou d'hygiène durant la séance ou lors de la phase de cicatrisation.

Les patients avec une dermatose chronique doivent être prévenus des possibles risques de localisation de leur dermatose sur tatouage.

Une éruption sur tatouage peut révéler une sarcoïdose.

Tout patient avec une pathologie chronique et/ou une immunodépression doit prendre contact avec son médecin pour discuter de la possibilité de se faire totouer. 
D of exogenous pigments and/or colorants into the dermis to produce a permanent design [1]. It is a global and ancient practice that has endured until now [2]. For the past twenty years, it has gained tremendous popularity especially among the young. Approximately 10 to $20 \%$ of the general population in Western countries is tattooed $[3,4]$. A similar prevalence of $10 \%$ was found in 2010 during a phone survey in France, with a peak of 20\% among the 24-35 years-old in France [5]. According to the Syndicat National des Artistes Tatoueurs, in 2013, there was approximately 2000 to 3000 "professional" tattooists in France, so 1 tattooist for 22,600 inhabitants [3,4]. However, the overall number of tattooists in France, including the unofficial ones, also called "scratchers" or "backyard tattooists", is unknown. Tattooing concern mainly a generation of men and women born after 1977 [3,4]. It is still a "young" generation that will live with their tattooed skin for the next 50 years. The introduction of exogenous pigments in the dermis is not totally harmless. Complications after tattooing have been recorded since the end of the 19th century [6-11]. Furthermore, medical progresses, high technology and evolution of fashion in tattooing have influenced the pattern of complications through times [12]. Even though the overall number of complications appears rare considering the millions of tattooed individuals throughout the world, a good knowledge of this practice is nowadays mandatory for any physician to allow him not only to manage adequately potential complications on tattoos but also provide correct advices to patients willing to get a tattoo. This review will focus on the cutaneous and extra-cutaneous complications after tattooing. We will not review the issue related to tattoo removal.

\section{Tattoo inks in 2016}

Most of the inks consist of a complex mix of organic or inorganic pigments dispersed in water plus additives to obtain ready-touse tattooing products (figure 1) $[13,14]$. Issues regarding tattoos inks include the presence of potentially genotoxic polycylic aromatic hydrocarbons in black colors, the use of organic colorants that have never been tested for the use in contact with the human body, the detection of heavy metallic salts as contaminants and the existence of nanoparticles [13]. In Europe, the council of Europe adopted a resolution in 2008 (ResAP(2008) 1) on requirements and criteria for the safety of tattoos and permanent make-up (PMU) [15]. This resolution applies to the composition and labelling of products used for tattoos and PMU; the risk evaluation required before products used for tattoos and PMU are placed on the market; the conditions of the application of tattoos and PMU; the obligation to inform the public and the consumer of the health risks of tattoos and PMU and tattooing practices. Several national authorities have used the resolution as the backbone of their regulations. Nowadays, most inks being used for tattoos are manufactured outside of Europe (with an estimation of 70-80\%). It is said that American products are used by professional artists, while Asian ones are distributed by non-professionals. Manufacturers in Europe have reinforced their inks and avoid ingredients that are banned for use in tattooing according to ResAP(2008)1. Even though the resolution is imperfect, it is a first step towards safer inks. The problems, ink manufacturers have to face nowadays, are the lack of harmonized analytical methods, of guidelines for risk assessment and of guidelines for good manufacturing practice [16].

\section{Cutaneous complications}

The frequency of the cutaneous complications related to tattooing remains globally unknown. Its prevalence varies greatly between $2 \%$ [17] to $27 \%$ [18], depending on the study settings (often self-reported questionnaires performed on internet, by phone or by direct interview) and the inquired symptoms, without a physical examination. Almost a third of the tattooed individuals report minor symptoms (itch, swelling) on one of their tattoos beyond 3 months after tattooing [18].

Cutaneous complications may be classified in various ways:

- according to the length of their evolution: acute and chronic reactions;

- according to the delay of onset after tattooing (early during the healing phase or delayed, after tattoo healing);

- or according to the type of reaction: infection, hypersensitivity reaction, etc.

None of these classifications is perfect as there can be some overlap between reactions and delay after tattooing. The latter classification, which is a clinical and pathological one, appears to be the easiest and the most convenient to us.

\section{Acute complications after tattooing}

Individuals who are getting tattooed always experience immediate, but transient, reactions during the procedure and the healing phase $[19,20]$. An acute aseptic inflammatory reaction of variable intensity with erythema, induration and an edematous "peau d'orange" with dilatation of the hair follicles of the tattooed skin develops immediately during the session. The fresh tattoo is usually tender surrounded by erythematous borders and the lines of the drawings are palpable. An ecchymotic purpura, and sometimes an underlying hematoma, may sometimes be visible. The tattoo heals within 2 to 3 weeks with superficial crusts and the ink retained in the epidermis is shed as the epidermis peels away. Such reactions occur to all tattooed individuals and should not be considered as "complications", but rather as belonging to the "natural history" of tattoos [20]. Disabling sterile edema may occur on the extremities of tattooed limbs. They require simple rest [21]. Acute transient lymphadenopathy of the tattoo draining area may be palpated during the healing phase. Acute contact dermatitis to any topical agent applied during the healing phase (disinfectant, healing 


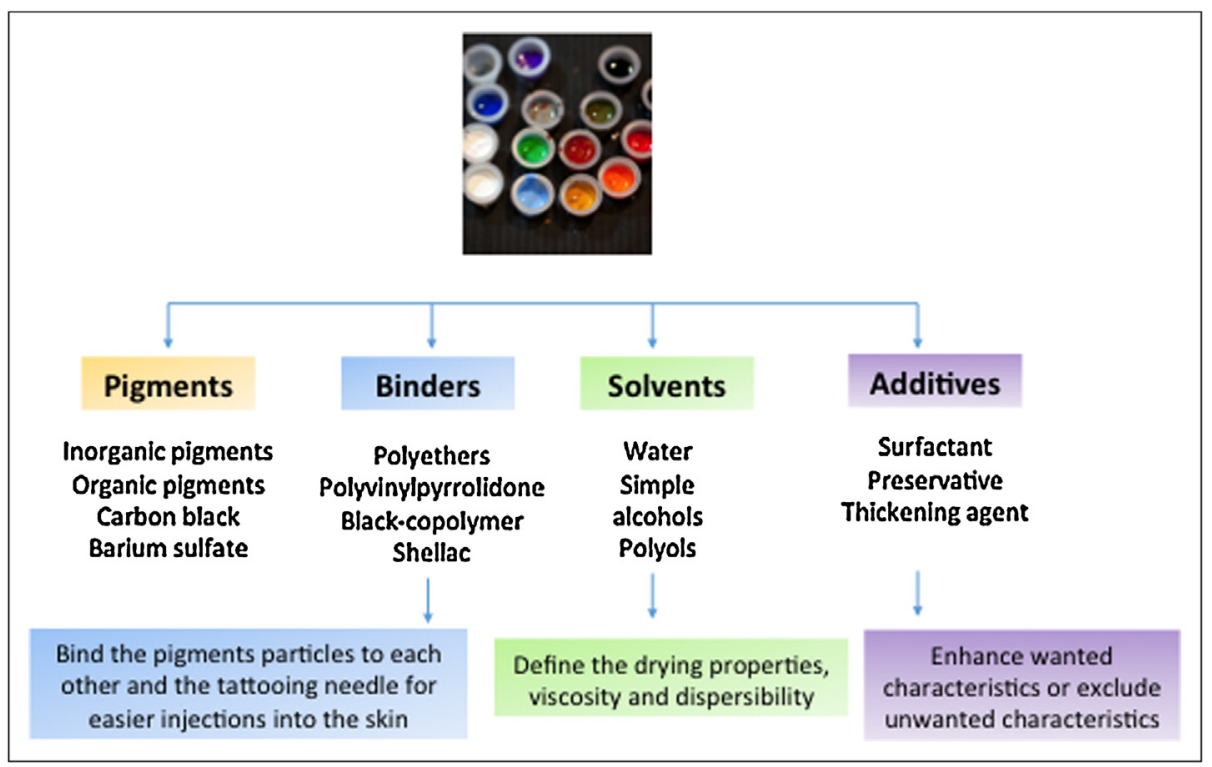

FIGURE 1

Composition of a tattoo ink (according to Dirks [14])

ointments such as dexpanthenol...) may occur in sensitized individuals (figure 2). Acute pyogenic bacterial infection can occur quickly in the case of lack/mistake during the procedure or lack of proper after-care during the healing phase. Tattoo pigment can spread in the superficial subcutaneous fat and be responsible for a "blurry halo" surrounding the tattoo after its completion. Such condition is called "tattoo blow-out" and may

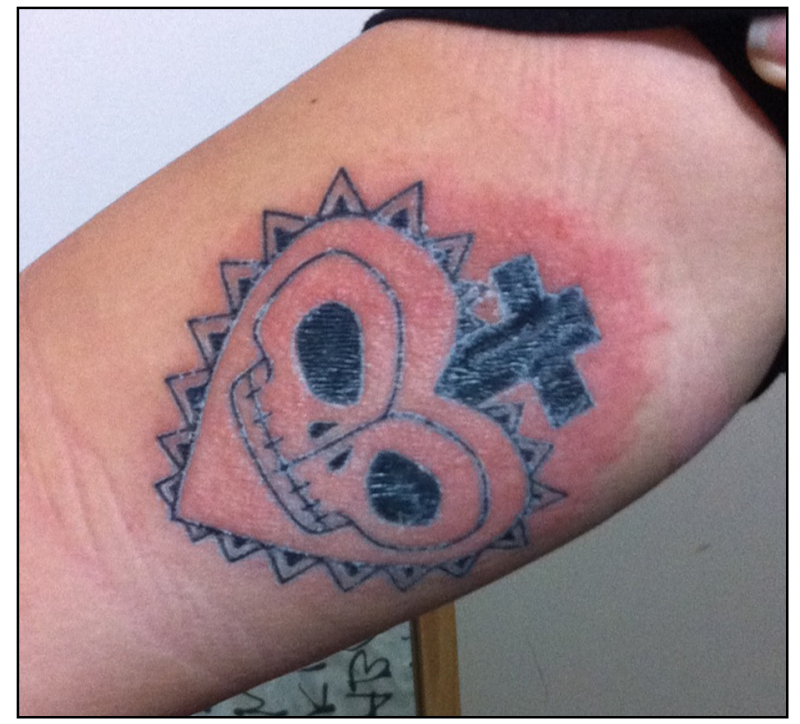

FIGURE 2

Contact eczema during the healing phase due to homeoplasmine cream (collection Dr L. Vesval Granville) be treated by laser (figure 3). "Tattoo blow-out" is the only "acute" complication that will remain permanent [22].

\section{Cutaneous infections}

Acute superficial and pyogenic infections (folliculitis, impetigo, ecthyma, furunculosis, erysipela, cellulitis) may occur after tattooing during the healing phase (figure 4) [23,24]. Infection can be restricted to a single color and should not be overlooked by considering wrongly an allergy reaction (figure 5). Gangrene, amputations and deaths were reported among sailors by the end of the 19th century [23]. Inoculation syphilis, the major complication of tattooing during the 19th century in Europe, has

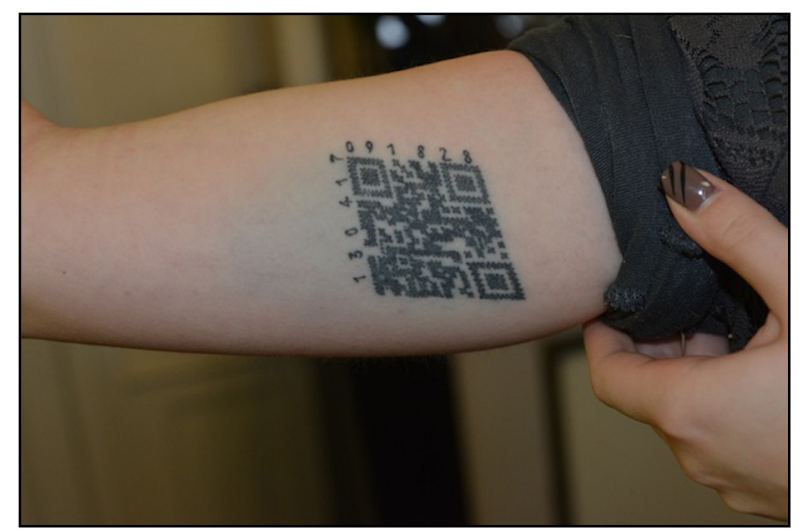

FIGURE 3

"Tattoo blow-out" on a recent black tattoo (collection Dr J.L. Rigon, Nancy) 


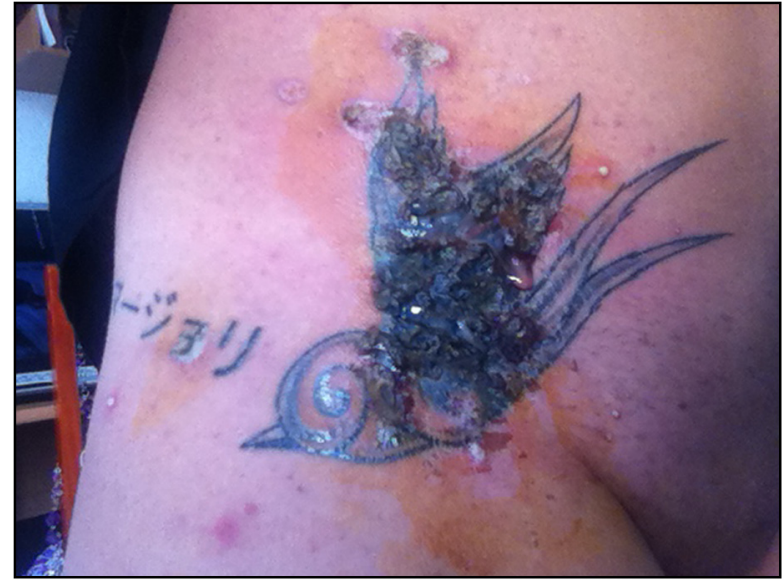

FIGURE 4

Pyogenic bacterial infection on a recent tattoo

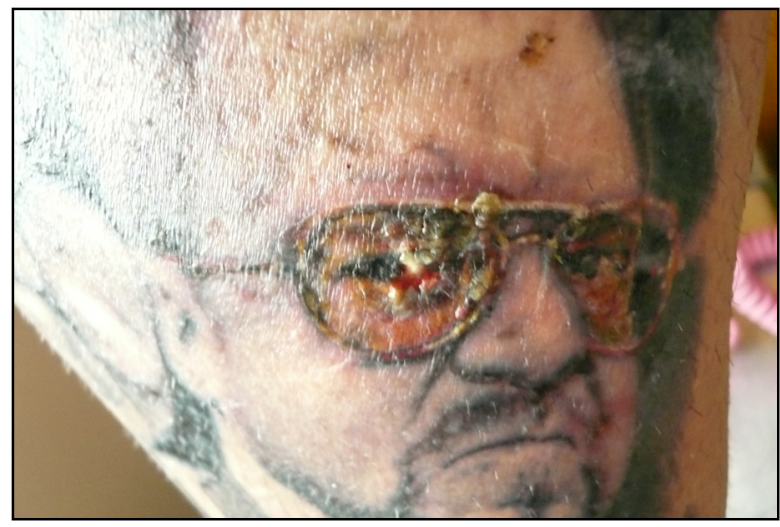

FIGURE 5

Infection restricted to the red part of a recent tattoo

now disappeared [6]. Hygiene measures, modern aseptic tattooing techniques and better education of the tattooists have participated in reducing such complications. However, unlicensed tattoo activity and asepsis can still lead to dramatic infection with cutaneous abscesses or necrotizing fasciitis.

Mycobacterial infections include: tuberculosis, leprosy and rapidly growing mycobacterias. Cutaneous tuberculosis is rare nowadays. Inoculation leprosy is restricted to India, a high endemic country for leprosy. Rapidly growing mycobacterial infections, especially with Mycobacterium chelonae infection, have emerged for the past years. Several outbreaks in tattoo parlors have been reported in France, in the USA and in Australia. Lesions are unspecific (chronic papules, pustules, lichenoid plaques, plaques with scales) and occur usually within one to three weeks after procedure (figure 6). Use of tap water mixed with the ink by the tattooist may be the cause of inoculation. Such

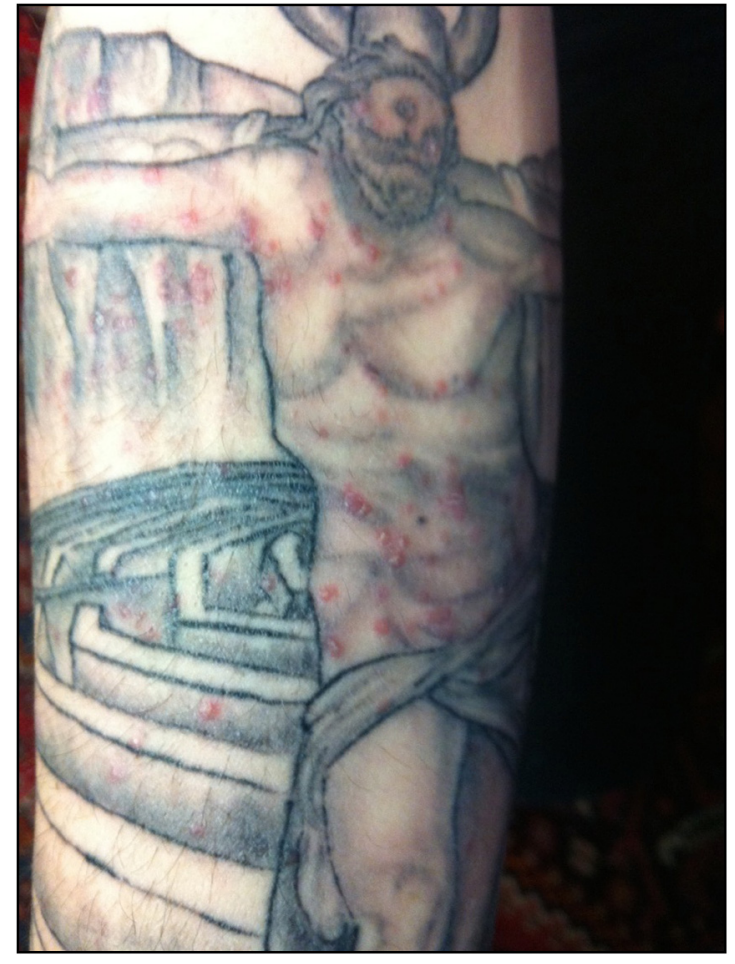

FIGURE 6

Mycobacterial infection on a recent tattoo. Notice that the lesions are restricted to the gray shadings only and not to the black lines (Dr AM Dournon, La Garenne Colombes)

complication should be evoked in case of multiple cases originating from the same tattooist/tattoo shop during a given time frame. Skin biopsies and bacterial cultures of skin and inks should confirm the diagnosis $[25,26]$.

Viral warts and molluscum contagiosum have been reported on tattoos. Skin lesions occur from one month to 10 years after tattooing, with variable numbers and size and may be restricted to one color. The mechanism of occurrence of the lesions remains unclear (figure 7).

Numerous cutaneous infections have been reported as case reports and are summarized in table $I$.

\section{Hypersensitivity reactions/allergies to tattoo pigments and dyes}

Introduction of exogenous pigments and dyes during tattooing may trigger a wide range of cutaneous reactions with histological patterns ranging from eczematous and lympho-histiocytic reactions (figure 8) to more "organized" pattern such as lichenoid, granulomatous foreign body (figure 9) or sarcoidosis-like reactions and pseudolymphoma $[20,27]$. They are from far the main cause of concern related to permanent tattooing nowadays $[18,28-30]$. Delay is highly variable ranging from immediately to 45 years after, usually several weeks to several years 


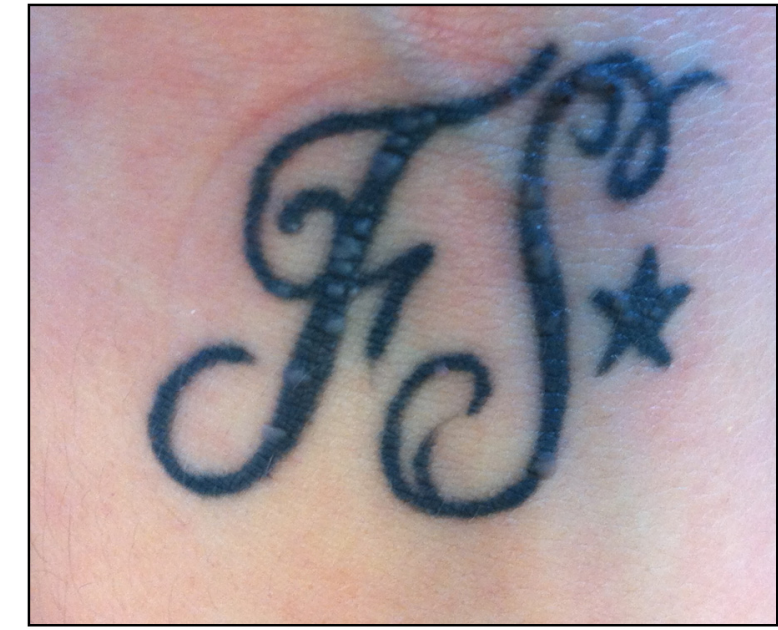

FIGURE 7

Viral warts restricted to tattooed areas (Dr S. Reberga, Toulouse)

TABLE I

Acute and chronic infectious complications on tattoos

Pyogenic infections

Folliculitis, furonculosis, erysipela, necrotizing fasciitis, gangrene, death

\begin{tabular}{l}
\hline Atypical germs \\
\hline Rapidly growing mycobacteria; cutaneous tuberculosis; leprosy; \\
syphilis (historical); tetanos; chancroid; diphteria \\
\hline Viral infection \\
\hline Warts; molluscum contagiosum; herpes simplex \\
\hline Fungal and parasitic infections \\
\hline Dermatophytia; leishmaniasis; aspergillosis; zygomycosis; \\
sporotrichosis; mycetoma
\end{tabular}

after tattooing. Red and purple/violet are the most common color involved, but reactions have been described with almost all colors, except white. Symptoms are non-specific, including tenderness, swelling, asymptomatic or itchy papules or nodules, isolated pruritus, and complete infiltration/induration of the color (figure 10). Necrosis occurs very rarely. Photosensitivity may be associated or even the only symptom [28]. The itch is often severe and may impact the quality of life of the patients [31]. Interestingly, in most of the cases, we observed reactions only on "small" sized tattoos and never on extensive tattoos. A precise diagnosis, made by the histopathological examination of a punch skin biopsy specimen, is mandatory. Any granulomatous reaction should prompt to look for underlying idiopathic sarcoidosis whereas a lichenoid reaction can be associated with genuine cutaneous or mucous lichen planus.

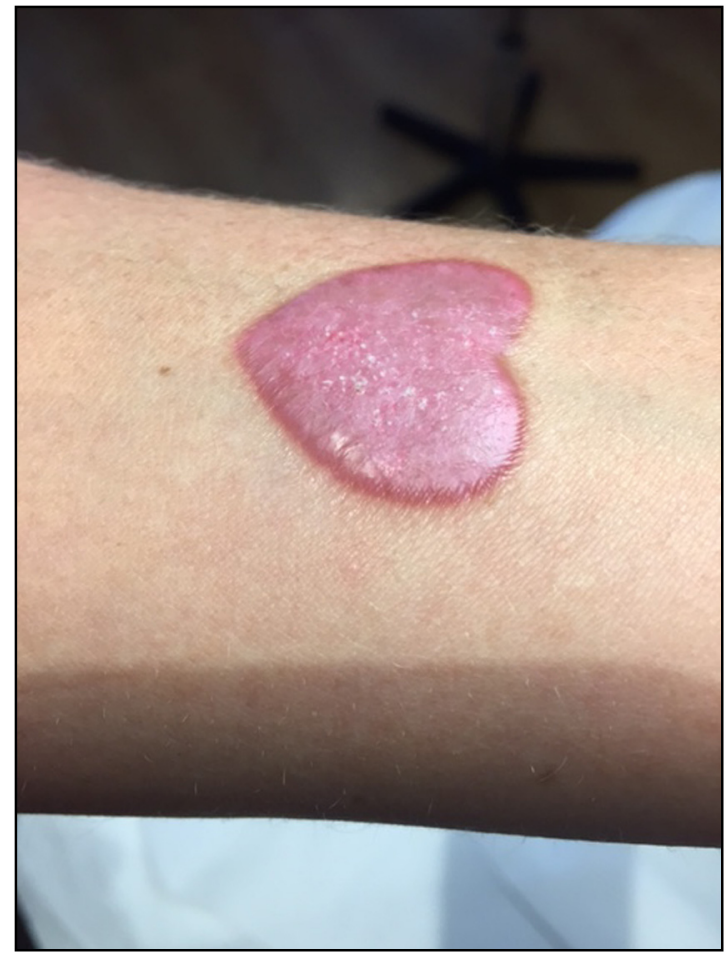

FIGURE 8

Lympho-histiocytic reaction towards the pink color (Dr S. Bosonnet, Montpellier)

Composition of elements in tattoo inks varies greatly, even among like-colored pigments. Epicutaneous tests are useless because often negative [32]. It may be related to limited transcutaneous absorption of the ink, but also because the culprit component may appear de novo in the skin. If a patient experienced a color specific tattoo reaction, he should be discouraged from getting tattooed with the same color - even if the ink brand is not the same. Moreover, he should be warned of the potential risk of reaction to other color(s) with close tone due to a possible common substance in both inks. Tattoo tests with a small amount of ink should be discouraged as it has no predictive value in the absence of standardized protocol and it may even trigger a sensitization to the ink.

Hypersensitivity reactions can resolve spontaneously, remain active or wax and wane for years. In our experience, tattooed individuals seem to experience periodic episodes of skin reactions but seek for medical attention only if the reaction becomes disabling or severe. Treatment is often difficult and usually only temporary as long as the culprit ink is still present in the skin. Highly potent topical corticosteroid ointment, tacrolimus or intralesional corticosteroids can be proposed. If reaction continues, removal by surgical excision, dermatoma shaving or destruction by $\mathrm{CO2}$ or Q-switched Nd:YAG laser can be suggested [33]. In the last case, we suggest to perform the laser treatment 


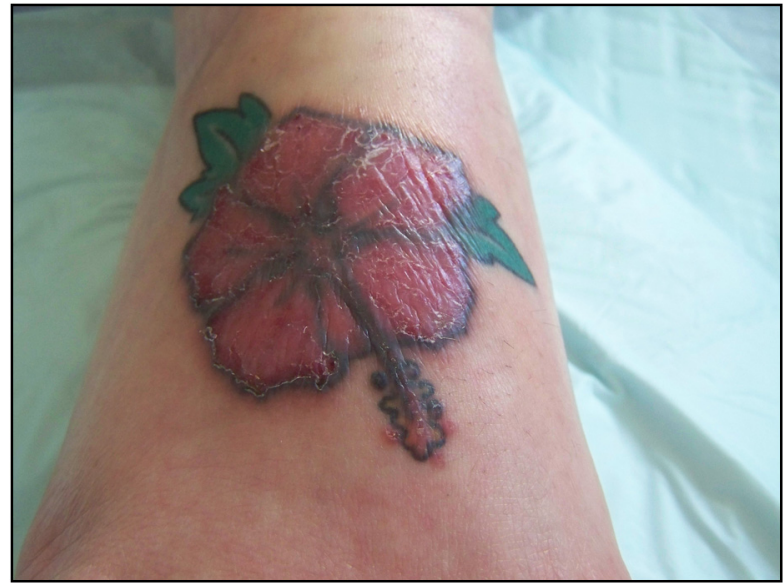

FIGURE 9

Granulomatous reaction on the red of a tattoo (Drs S. Hakimi and P. Del Giudice, Nice)

with ongoing corticosteroid treatment to prevent rare localized or generalized allergic reaction after laser treatment [34].

\section{Skin tumors arising on tattoos}

Cutaneous malignancies arising in tattoos have been reported for the past 40 years in the literature primarily melanoma, basal cell carcinoma and squamous cell carcinoma; melanoma being the most "frequently" reported [35]. They are still considered as a coincidental finding nowadays [35]. There is a potential risk that a malignant lesion develops coincidentally at the same location as a tattoo, preventing from an early diagnosis and management. Clinical and dermatoscopic surveillance of naevus is more difficult

\begin{tabular}{l} 
TABLE II \\
Tumors arising on tattoos \\
Malignancies \\
\hline Melanoma \\
\hline Basal cell carcinoma \\
\hline Squamous cell carcinoma \\
\hline Isolated or multiple keratoacanthoma \\
\hline Pseudoepitheliomatous hyperplasia \\
\hline Anecdotal tumor: cutaneous lymphoma, Darier-Ferrand \\
dermato-fibrosarcoma, leiomyosarcoma \\
\hline Benign tumors \\
\hline Seborrheic keratosis \\
\hline Histiocytofibroma \\
\hline Lipoma
\end{tabular}

in case of tattooing (figure 11). Tattooing over a benign melanocytic naevus may trigger its sudden clinical change that lead to surgical removal and pathological examination to distinguish between a traumatized naevus and malignant degeneration. Keratoacanthomas (KA) and pseudoepitheliomatous hyperplasia (PH) develop as a fast-occurring cutaneous reaction strictly to the part of tattoo, usually on the red color. Distinction between PH, KA and squamous cell carcinoma can be challenging and implies fullthickness biopsies and/or surgical removal of the entire lesion with thorough histological examination (table II) [36].
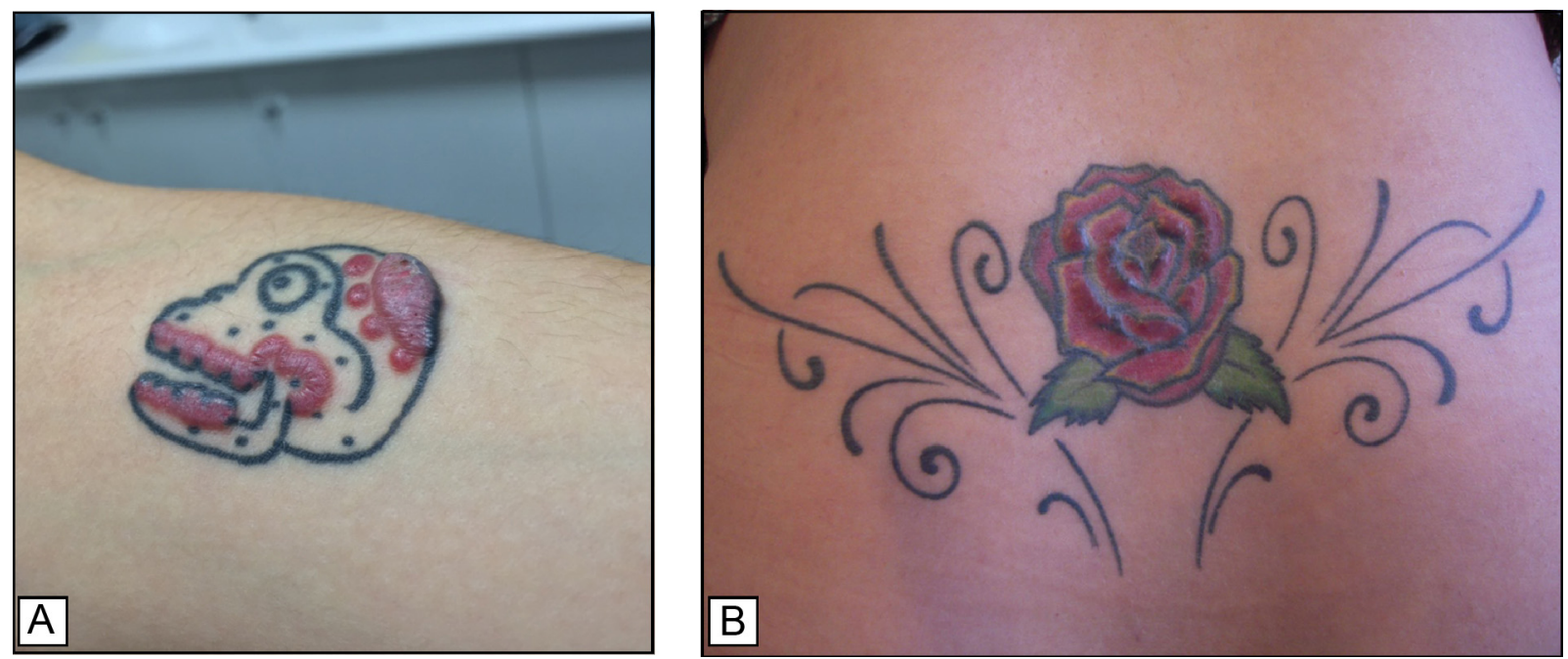

FIGURE 10

Different clinical presentation of red tattoo reactions. A. A typical infiltration restricted to all the red parts of a tattoo highly evocative of an allergy to red (Dr X. Lemercier, Vouneuil-sur-Vienne). B. Scattered infiltrated papules in the red of a tattoo (Dr 0. Rigoulet, Pau) 


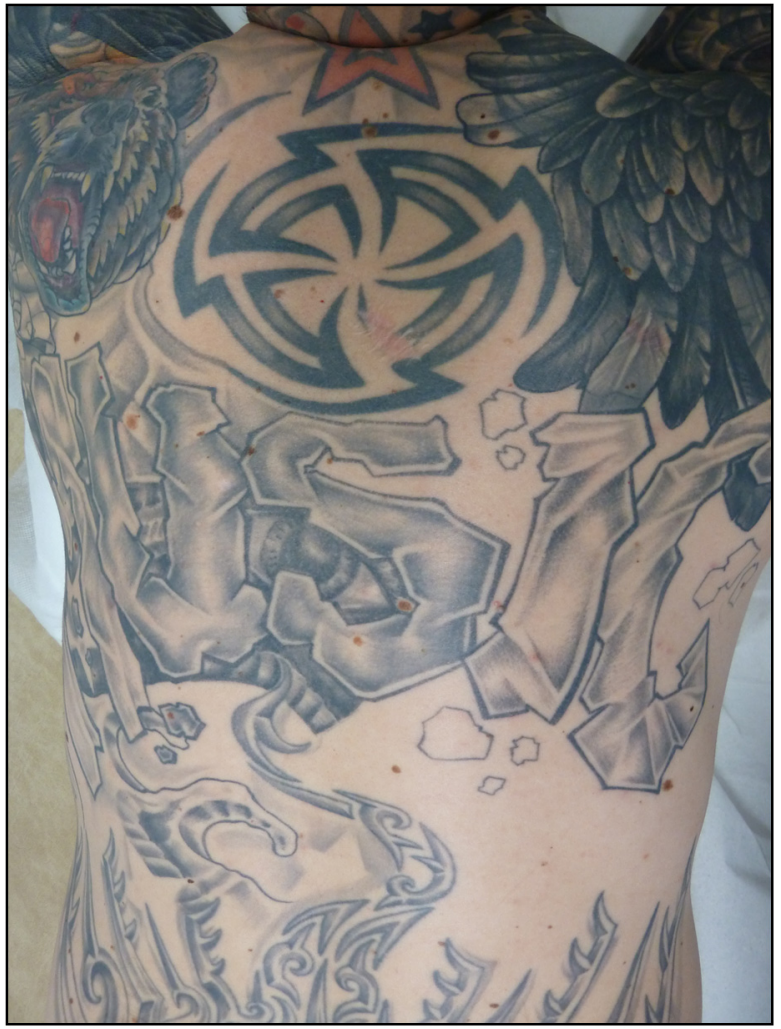

FIGURE 11

A patient with extensive tattoos on the whole back making it difficult to examine numerous moles

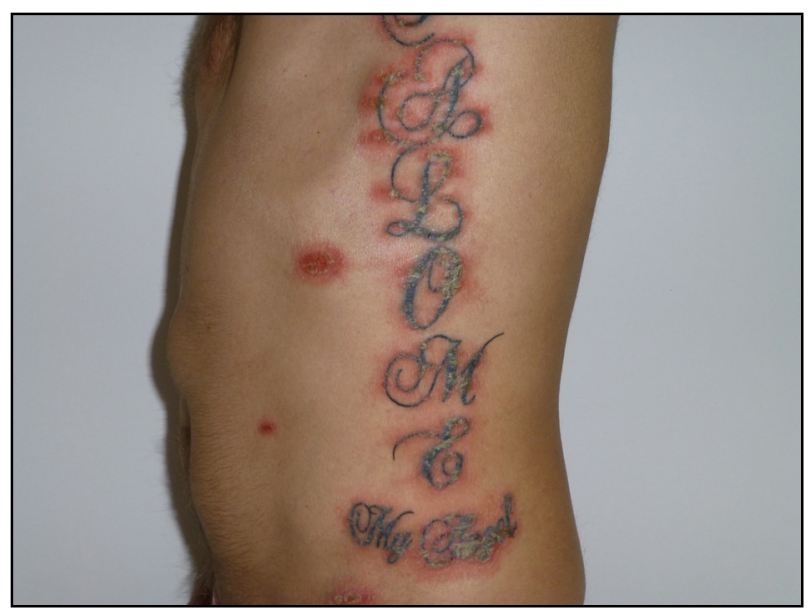

FIGURE 12

Psoriasis on a tattoo (Dr E. Estève, Orléans)
TABIE III

Dermatoses reported on tattoos

Sarcoidosis

Psoriasis

Cutaneous lupus (discoid or subacute types)

Vitiligo

Cutaneous vasculitis

Lichen planus

Lichen sclerosus

Darier's disease

Perforating dermatoses

Granuloma annulare

Lipoidic necrobiosis

Miliums

Epidermoid cysts

Pyoderma gangrenosum

Traumatized naevus by a tattoo

Skin burns and cheloids after laser hair removal on a tattooed area

\section{Localization of skin disorders to tattoo}

The number of dermatoses that can develop within a tattoo is plentiful (table III) [7-11]. Individuals with a chronic skin disease, which are known to koebnerize, should be warned of the potential risk of localization of the skin disease on a tattoo, if the dermatosis is active especially. They include mainly psoriasis, vitiligo or lichen planus (figure 12). Koebner phenomenon was initially described in patients with psoriasis. The risk of localization of psoriasis in tattoos is related to the genetic background of the individual and the activity level of the disease at the time of tattooing. The patient should know that at any time of his life, a lesion may occur on a tattoo, fortuitously or not. Sarcoidosis on tattoos has been known for years. Granulomatous reaction to tattoos, restricted to one color or not, may reveal or accompany systemic sarcoidosis (figure 13). Cases of cutaneous sarcoidosis restricted to one color of pigment raise the question of a true sarcoidal hypersensitivity reaction to the exogenous pigment or the first manifestation of a systemic disease. Any granulomatous reaction should prompt to look for sarcoidosis. The presence of other cutaneous lesions or extra-cutaneous granulomas helps in distinguishing sarcoidosis from a hypersensitivity reaction [37]. Various cutaneous complications have been reported in anecdotal case reports: pyoderma gangrenosum, vasculitis, perforating dermatosis, granuloma annulare, Darier's disease, erythema multiforme. . . Burns and keloids may occur if laser hair 


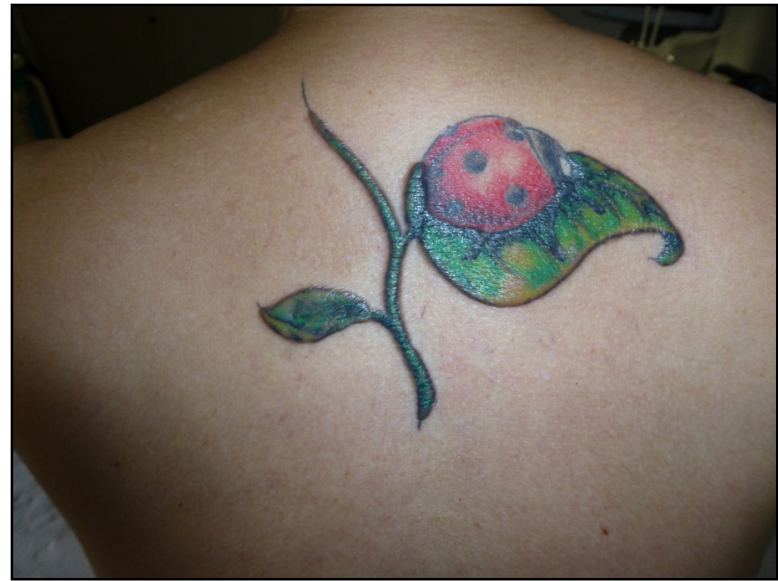

FIGURE 13

Sarcoidosis on a tattoo (Dr E. Estève, Orléans)

removal is performed on tattooed areas. The management of dermatoses on tattoos does not differ from plain skin.

\section{Extra-cutaneous complications}

The risk of transmission of hepatitis C, hepatitis B and HIV is feared because of the suffusion of blood that happens during the tattooing procedure and the use of needles. The first outbreak of hepatitis in a tattoo parlor war reported in the 50 s. Nowadays, if the rules of hygiene and asepsis are respected, the material sterilized and if no needle is shared between customers, the risk of infection is close to nil [38]. The risk remains high in any other situations where sterility and hygiene are not guaranteed (prison, home-tattooing, traditional tattoos in third world countries).

Sepsis are exceptional nowadays. They can occur in case of immunosuppression [39]. A few cases of endocarditis prompt us to suggest that patients with prior heart conditions should be referred to cardiologists before tattooing for advice [40].

Cases of uveitis associated with granulomatous tattoo reactions have been reported for the past years by the ophthalmologists [41]. It is unclear if it is a genuine distinct entity or a variant of sarcoidosis.

The migration of tattoo pigments to draining lymph nodes is constant. It has not been established clinically that this migration lead to an impairment of the lymph node function. Virtually, all the tattooed individuals have lymph nodes with pigments that will remain all life long. Sometimes, local lymph nodes can be palpated, up to $3 \mathrm{~cm}$, sometimes just after tattooing and sometimes later on [42]. The diagnosis of reactional tattooed lymph node is a diagnosis of elimination. Unfortunately, in case of asymmetric lymph node in a young patient, especially in case of past history of cancer, a biopsy is mandatory. Lymph nodes can be discovered by accident during imaging (CT scan, echography....), during PET scanning (abnormal intake of 18fluorodeoxyglucose) or during a surgical procedure or a lymph node dissection. Hyperdensities can be seen on the CT scanner and hyperechogenicities during an ultrasound exploration. The discovery of pigmented black lymph node may be problematic during a sentinel lymph node procedure. Macroscopically, the lymph node appears grayish-black, but the histopathological analysis will confirm its benign nature with reactive follicular hyperplasia and sinus histiocytosis and histiocytes containing pigment. Tattoos on a draining lymph node area should always be mentioned for the pathologist in case of lymph node biopsy or dissection. Lastly, pseudo-axillary calcifications during mammography have been reported several times [43].

At last, a certain number of anecdotal complications have been reported in the literature after tattooing without any clear link of causality thus far (neuropathic pain, brachial plexopathy, interstitial pneumopathy, psoriatic arthritis...).

\section{Conclusions}

Tattooing can result in a wide variety of complications, whose incidence is unclear right now. Infections are nowadays directly related to the lack of asepsis and hygiene during the tattooing procedure and can be avoided by education and training of tattoo professionals. Patients with a known cutaneous disease should be warned of a potential risk of localization of their disease to the tattoo. A skin eruption restricted to a tattoo may reveal sarcoidosis. Hypersensitivity reactions to tattoo pigments are not predictable. Therefore, regulatory control over ink manufacturing is important to avoid introduction of toxic, carcinogenic and/or immunogenic products. However, despite any

\begin{tabular}{l} 
TABLE IV \\
At risk situations when a tattoo should be discussed with the \\
treating physician or even contraindicated [44] \\
Past history of tattoo reaction with a specific color ${ }^{1}$ \\
\hline Personal history of melanoma \\
\hline Familial history of melanoma \\
\hline Atypical mole syndrome \\
\hline Past history of immunosuppressive conditions (leukaemia, HIV...) \\
\hline Intake of immunosuppressive treatment(s) \\
\hline Cardiopathy (endocarditis risk) \\
\hline Inherited or acquired blood disorders (hemophilia, Von Willebrand...) \\
\hline Pregnancy ${ }^{2}$ \\
\hline Breast feeding ${ }^{2}$
\end{tabular}

${ }^{1}$ Contraindication for a tattoo with the same color or colors with close tone. ${ }^{2}$ Strict contraindications for the author. 
control measures that may be instituted, complications will still occur.

Besides, patients with chronic conditions and/or impaired immunity should discuss with their physician about the possibility and when to have a tattoo (table IV).

Lastly, one should not forget that the most common risk of tattooing remain regrets and an undesirable ugly tattoo.

\begin{abstract}
Acknowledgments: The author wishes to thank all the dermatologists who kindly accepted to share the pictures of their personal cases of tattoo reactions.
\end{abstract}

Disclosure of interest: the author declares that he has no competing interest.

\section{References}

[1] Goldstein N. Tattoos defined. Clin Dermatol 2007;25:417-20

[2] Deter-Wolf A, Robitaille B, Krutak L, Galliot S. The world's oldest tattoos. Journal of Archaeological Science: Reports 2016;5:19-24.

[3] Kluger N. Epidemiology of tattoos in industrialized countries. Curr Probl Dermatol 2015:48:6-20

[4] Kluger N. Tatoués, qui êtes-vous ? Caractéristiques démographiques et comportementales des personnes tatouées. Ann Dermatol Venereol 2015;142:410-20.

[5] Fourquet J. Les français et les tatouages. IFOP; 2010 [http://www.ifop.com/?option= com_publicationgtype $=$ pollgid $=1220]$.

[6] Kluger N, Guillot B. Les annales de dermatologie et de syphiligraphie en 1895: chancres syphilitiques multiples consécutifs au tatouage. Ann Dermatol Venereol 2009;136:393-4.

[7] Beerman H, Lane RA. Tattoo; a survey of some of the literature concerning the medical complications of tattooing. Am 」 Med Sci 1954;227:444-64

[8] Davis RG. Hazards of tattooing: report of two cases of dermatitis caused by sensitisation to mercury (cinnabar). U S Armed Forces Med J 1960;11:261-80

[9] Goldstein N. IV. Complications from tattoos. J Dermatol Surg Oncol 1979;5:869-78.

[10] Kluger N. Cutaneous complications related to permanent decorative tattooing. Expert Rev Clin Immunol 2010;6:363-71.

[11] Sperry K. Tattoos and tattooing. Part II: gross pathology, histopathology, medical complications, and applications. Am J Forensic Med Pathol 1992;13:7-17.

[12] Kluger N, Bessis D, Raison-Peyron N, Guillot B. Tatouages permanents: de nouvelles complications au $x \times 1^{e}$ siècle. Presse Med 2006;35:1598-600.

[13] Laux P, Tralau T, Tentschert J, Blume A, Dahouk SA, Bäumler W, et al. A medicaltoxicological view of tattooing. Lancet 2015 http://dx.doi.org/10.1016/S0140-6736(15) 60215-X [pii: S0140-6736(15)60215-X].
[14] Dirks M. Making innovative tattoo ink products with improved safety: possible and impossible ingredients in practical usage. Curr Prob Dermatol 2015;48:118-27.

[15] Resolution ResAP(2008)1 on requirements and criteria for the safety of tattoos and permanent make-up (superseding Resolution ResAP(2003)2 on tattoos and permanent make-up), https://wcd.coe.int/ViewDoc. jsp?id $=1254065$

[16] Michel R. Manufacturing of tattoo ink products today and in future: Europe. Curr Prob Dermatol 2015;48:118-27.

[17] Kazandjieva J, Tsankov N. Tattoos: dermatological complications. Clin Dermatol 2007;25: 375-82.

[18] Høgsberg T, Hutton Carlsen K, Serup J. High prevalence of minor symptoms in tattoos among a young population tattooed with carbon black and organic pigments. J Eur Acad Dermatol Venereol 2013;27:846-52.

[19] Kluger N. Acute complications of tattooing presenting in the ED. Am J Emerg Med 2012:30:2055-63.

[20] Kluger N, Plantier F, Moguelet P, Fraitag S. Tattoos: natural history and histopathology of cutaneous reactions. Ann Dermatol Venereol 2011;138:146-54 [quiz 144-5, 155].

[21] Kluger N, Hubiche T, Del Giudice P. Tattooinduced edema of the lower limbs mimicking cellulitis: report of two cases. Int J Dermatol 2013;52:384-6.

[22] Kluger N. Blurry halos around tattoos: a new case of "tattoo blow-out". Int J Dermatol 2014:53:e44-6.

[23] Long GE, Rickman LS. Infectious complications of tattoos. Clin Infect Dis 1994;18:610-9.

[24] Kluger N. Cutaneous infections related to permanent tattooing. Med Mal Infect 2011; 41:115-22

[25] Kluger N, Muller C, Gral N. Atypical mycobacteria infection following tattooing: review of an outbreak in 8 patients in a French tattoo parlor. Arch Dermatol 2008;144:941-2.
[26] Kennedy BS, Bedard B, Younge M, Tuttle D, Ammerman E, Ricci J, et al. Outbreak of Mycobacterium chelonae infection associated with tattoo ink. N Engl J Med 2012;367: 1020-4.

[27] Høgsberg T, Thomsen BM, Serup J. Histopathology and immune histochemistry of red tattoo reactions. Skin Res Technol 2015;21: $449-58$.

[28] Hutton Carlsen K, Serup J. Photosensitivity and photodynamic events in black, red and blue tattoos are common: a "Beach Study". J Eur Acad Dermatol Venereol 2014;28:231-7.

[29] Brady BG, Gold H, Leger EA, Leger MC. Selfreported adverse tattoo reactions: a New York City Central Park study. Contact Dermatitis 2015;73(2):91-9.

[30] Kluger N. Self-reported tattoo reactions in a cohort of 448 French tattooists. Int J Dermatol 2015. http://dx.doi.org/10.1111/ijd.13030.

[31] Hutton Carlsen K, Serup J. Patients with tattoo reactions have reduced quality of life and suffer from itch: Dermatology Life Quality Index and Itch Severity Score measurements. Skin Res Technol 2015:21:101-7.

[32] Serup J, Hutton Carlsen K. Patch test study of 90 patients with tattoo reactions: negative outcome of allergy patch test to baseline batteries and culprit inks suggests allergen (s) are generated in the skin through haptenization. Contact Dermatitis 2014:71:255-63.

[33] Kluger N. Réactions dites " allergiques » sur tatouage : prise en charge et algorithme thérapeutique. Ann Dermatol Venereol 2016 [under press].

[34] Zemtsov A, Wilson L. CO2 laser treatment causes local tattoo allergic reaction to become generalized. Acta Dermatol Venereol 1997:77:497.

[35] Kluger $N$, Koljonen $V$. Tattoos, inks, and cancer. Lancet Oncol 2012:13:e161-8.

[36] Kluger N, Durand L, Minier-Thoumin C, Plantier F, Cotten H, Berteloot E, et al. Pseudoepitheliomatous epidermal hyperplasia in tattoos: 
report of three cases. Am I Clin Dermatol 2008;9:337-40.

[37] Kluger N. Sarcoidosis on tattoos: a review of the literature from 1939 to 2011. Sarcoidosis Vasc Diffuse Lung Dis 2013;30:86-102.

[38] Urbanus AT, van den Hoek A, Boonstra A, van Houdt R, de Bruijn LJ, Heijman $T$, et al. People with multiple tattoos and/or piercings are not at increased risk for HBV or HCV in The Netherlands. PLoS One 2011;6: e24736.
[39] Tendas A, Niscola P, Barbati R, Abruzzese E, Cuppelli L, Giovannini $M$, et al. Tattoo related pyoderma/ectyma gangrenous as presenting feature of relapsed acute myeloid leukaemia: an exceptionally rare observation. Injury 2011;42:546-7.

[40] Kluger N. Bacterial endocarditis and body art: suggestions for an active prevention. Int Cardiol 2009:136:112-3.

[41] Ostheimer TA, Burkholder BM, Leung TG, Butler NJ, Dunn JP, Thorne JE. Tattoo-associated uveitis. Am ] Ophthalmol 2014:158 [637.e1-43. e1].

[42] Kluger N, Cohen-Valensi R, Nezri M. Black lymph nodes-and a colourful skin. Lancet 2008;371:1214

[43] Kluger N. Tatouages et imagerie médicale problèmes et mythes. Presse Med 2014:43 529-33.

[44] Kluger N. Contraindications for tattooing. Cur Probl Dermatol 2015;48:76-87. 